\title{
Immunotherapy for Visceral Leishmaniasis: Ability of Factors Produced during Anti-leishmania Responses of Skin Test Positive Adults to Inhibit Peripheral Blood Mononuclear Cell Activities Associated with Visceral Leishmaniasis
}

\author{
Bettie J Holaday \\ Núcleo de Medicina Tropical, Universidade Federal do Ceará, Rua Alexandre Baraúna 949, 60430-160 \\ Fortaleza, CE, Brasil
}

\begin{abstract}
The course of human Leishmania chagasi infections appears to be determined by the balance between type 1 (T1) CD4+ and CD8+T suppressor (Ts) cell activities. Skin test positive adults living in hyperendemic areas who have no history of visceral leishmaniasis (VL) have T1 CD4+ T cell immunodominant responses against $\mathrm{L}$. chagasi. The cytokines they secrete during anti-leishmania responses are a probable source of cytokines which inhibit the CD8+Ts cells associated with VL. The ability of supernatants generated from peripheral blood mononuclear cells derived from skin test positive adults to reverse immune responses which appear to be mediated by CD8+Ts cells was assessed in three sets of screening assays. The supernatants displayed three candidate factors. One, which could be explained by Leishmania antigens in the supernatant, decreased high endogenous IL-10 secretion characteristic of one class of VL patients. A second activity decreased high endogenous proliferation characteristic of the same class of patients without decreasing antigen specific proliferation. The third activity inhibited or killed CD8+T cells but not CD4+T cells. These activities might be useful in treating $V L$.
\end{abstract}

Key words: leishmaniasis - CD8+ T suppressor (Ts) - immunotherapy - interleukin (IL)-10 - IL-12 - antigens

Humans with a history of infection with Leishmania chagasi display a continuum of immune responses to the parasite. Subjects who have lived in hyperendemic areas for many years without clinically evident infections who have strong DTH+ responses against Leishmania are thought to have the strongest protective immune responses. They have the strongest Leishmania specific T1 CD4+ T cell responses (Holaday et al. 1993a). Conversely, CD4+ T cells are depleted in visceral leishmaniasis (VL) patients especially in the spleen (Cenini et al. 1993). CD8+ T cells, but not CD4+ $\mathrm{T}$ cells, isolated from patients with VL inhibit Leishmania specific proliferation and IFN-gsecretion while increasing (interleukin) IL-10 secretion when mixed with autologous peripheral blood

\footnotetext{
This work was supported by United States National Institutes of Health grants 5PO1 AI-26512 and AI-26918 and TDR Strengthening Grant 880-225.

Present address: 170A Grattan St., San Francisco, CA 94117, USA. Fax: +1-415-566-9776.

Received 22 October 1996

Accepted 8 July 1998
}

mononuclear cells (PBMC) isolated after successful treatment (Holaday et al. 1993a). CD4+ T cell lines cannot be isolated from acute VL patients until CD8+ T cells are removed by cell sorting. Patients who have been successfully treated for VL generally have more variable responses to $L$. chagasi antigens ranging from responses characteristic of acute patients to those of DTH+ adults (Holaday et al. 1993b). Thus, the course of L. chagasi infections appears to be due to the balance between $\mathrm{T} 1$ CD4+ T cells and CD8+ T suppressor (Ts) cells. These findings are in agreement with those of Uyemura et al. (1993) who found both T1 CD4+ and T2 CD8+ T cells in human cutaneous Leishmania lesions.

Recent attempts to identify cytokines which might be useful in treating VL have focused on cytokines thought to maintain the balance between $\mathrm{T} 1$ and T2 CD4+ T cells in spite of the lack of evidence for a role for T2 CD4+ T cells in symptomatic human $L$. chagasi and $L$. donovani infections (Hsieh et al. 1993, Manetti et al. 1993, Carvalho et al. 1994, Ghalib et al. 1995). The current study is the first to focus on detecting cytokines which might maintain a T1 CD4+ T cell immunodominant response by inhibiting the CD8+ Ts cells 
associated with symptomatic human L. chagasi infections. Cytokines with this property might restore the ability of VL patients' immune systems to eradicate Leishmania infections.

Three different types of screening assays were done. Each one provided different information. Since DTH+ adults have the strongest T1 CD4+ T cell response against $L$. chagasi the cytokines they secrete during anti-leishmania responses are the most probable source of cytokines which inhibit the CD8+ Ts cells associated with VL. In the first set of assays supernatants generated by DTH+ adult PBMC in the presence (DS+A) or absence (DS-A) of $L$. chagasi antigens were tested for their ability to convert VL patient PBMC responses thought to be mediated by CD8+ Ts cells, that is, to decrease IL10 secretion, increase IFN-g secretion, or increase Leishmania specific proliferation. The effects of DS+A were compared to those of IL-12 and antiIL-10 which are thought to favor T1 CD4+ T cell activity over T2 CD4+ T cell activity (Hsieh et al. 1993, Manetti et al. 1993, Carvalho et al. 1994, Ghalib et al. 1995). The second assay used a reconstitution assay to measure the ability of DS+A and IL-12 to prevent CD8+ Ts cell mediated changes in cytokine secretion and proliferation. The third set of assays directly tested the ability of a supernatant (CD4S) generated from Leishmania specific T1 CD4+ T cell lines derived from DTH+ adults to inhibit the growth of CD4+ and CD8+ T cell lines.

The three sets of assays identified three candidate activities, Leishmania antigens and two soluble factors. The assays also revealed the existence of two categories of VL patients, those with high and low levels of endogenous IL-10 secretion, which responded differently to immunomodulators.

\section{MATERIALS AND METHODS}

Human subjects - Subjects of the study were residents of the states of Ceará (CE), Piauí (PI), or Rio Grande do Norte (RN) in northeastern Brasil. $\mathrm{DTH}+$ adults from a hyperendemic area near Itapipoca, CE, were selected as representative of people with strong protective immune responses against $L$. chagasi (Evans et al. 1992). PBMC from five DTH+ adults (ages 16-64, mean $=33$ years) with indurations of 17-25 mm measured $48 \mathrm{hr}$ after intradermal injection of $L$. chagasi antigens were used to generate the DS-A and DS+A supernatants. Nineteen acute patients (ages 14-58, mean $=28$ years) were self referred to Hospital São José or Hospital Walter Cantído in Fortaleza, CE; Santa Casa in Sobral, CE; Hospital Gizelda Trigueiro in Natal, RN and the Infectious Diseases Hospital of the Federal University of Piauí in Terezina; or were identified in field studies conducted by Fundação
Nacional de Saúde. All diagnoses were confirmed by the presence of amastigotes in bone marrow aspirates. Patients, except P14 and P23, were successfully treated with pentavalent antimony $(20 \mathrm{mg} /$ $\mathrm{kg}$ /day) for 20 days. P14 had been symptomatic for eight months prior to seeking treatment. She showed no signs of clinical improvement after nine days of treatment with allopurinol, so she was then successfully treated with pentavalent antimony as described above. Blood for this study was drawn from P14 after allopurinol treatment was completed but before pentavalent antimonial treatment had begun. P23 had multiple relapses during a one and a half year period in spite of treatment with pentavalent antimony, allopurinol and splenectomy. The studies reported here were made during combined pentavalent antimony/allopurinol treatment of P23. All assays of acute VL patient PBMC were performed during the seven month period of AprilOctober. P2 was successfully treated for VL in 1990 and has been free of kala azar symptoms since the end of treatment.

Parasite antigens - L. chagasi promastigotes were cultured in HOSMEM II medium containing $20 \%$ fetal calf serum, washed three times in PBS, harvested from stationary phase cultures by centrifugation, resuspended in PBS and lysed by sonication or repeated freeze thawing (Berens \& Marr 1978). An aliquot of antigen suspension was assayed for protein content using a modification of the Lowry Rosebrough method (Dulley \& Grieve 1975). Antigens prepared in this way are not mitogenic to PBMC from non-immune subjects.

$P B M C$ cultures and generation of $D S+A$ and $D S-A$ - Assays of distilled water produced by the still in the Núcleo de Medicina Tropical showed the water to be contaminated with endotoxin so neither the water nor glassware washed or rinsed in it were used to prepare or store the media used in these assays. All reagents and plasticware used in preparing the media or which came into contact with blood or PBMC were prescreened for endotoxin contamination by the manufacturers. Iscove's modified Dulbecco's medium and Hank's balanced salt solution were prepared with a single lot of Gibco (Grand Island, NY) sterile, filtered, endotoxin prescreened water in a biosafety cabinet. Media were prepared in newly unwrapped, sterile plasticware and stirred with newly unwrapped, sterile pipettes. All PBMC assays were performed with sera from a single bottle of fetal calf serum (Hyclone, Logan UT) and a single collection of human $\mathrm{AB}+$ serum (collected from a healthy, fasting North American male donor). Media were filtered through 0.2 micron bottle top filters into sterile bottles which had contained Gibco water only moments before. Peripheral blood was collected 
by venipuncture. Mononuclear cells were separated by centrifugation at $200 \mathrm{~g}$ for $20 \mathrm{~min}$ at $20^{\circ} \mathrm{C}$ over ficoll-hypaque. Cells were washed twice in Hank's balanced salt solution containing $0.25 \%$ bovine serum albumin. The washed cells were cultured at $3 \times 10^{6}$ cells $/ \mathrm{ml}$ in Iscove's modified Dulbecco's medium supplemented with $10 \%$ fetal calf serum $/ 10 \%$ human $\mathrm{AB}+$ serum and containing antibiotic/antimycotic in the absence or presence of $20 \mathrm{mg} / \mathrm{ml}$ Leishmania antigens. Supernatants were collected on day 4, pooled, aliquotted and stored frozen prior to assay. Supernatants generated from DTH+ adult PBMC with or without $L$. chagasi antigens were designated DS+A and DSA, respectively. DS+A contained $1.9 \mathrm{ng} / \mathrm{ml} \mathrm{IFN-g}$ and $0.07 \mathrm{ng} / \mathrm{ml} \mathrm{IL-10.} \mathrm{DS-A} \mathrm{lacked} \mathrm{measurable}$ amounts of IFN-g but contained $0.07 \mathrm{ng} / \mathrm{ml}$ IL-10.

VL patients are anemic and leukopenic and the limited numbers of PBMC which could be collected from them prevented all assays from being performed with PBMC from each patient. PBMC from all 19 patients were assayed for proliferation but PBMC from only 12 patients were assayed for cytokine secretion. In addition, there were sufficient PBMC from only seven patients to allow testing of all the immunomodulators on proliferation and cytokine secretion. PBMC from P19 were collected and assayed twice, once three days after initiation of treatment and a second time 10 days after initiation of treatment (P19R).

Assays of the effects of immunomodulators on PBMC activities - In assays of PBMC from acute VL patients PBMC were cultured in triplicate, as described above. When immunomodulators were added to the cultures they were present at the following concentrations: rIL-12 $50 \mathrm{U} / \mathrm{ml}$ (Genetics Institute, Cambridge, MA), anti-IL-10 $30 \mathrm{mg} / \mathrm{ml}$ (DNAX, Palo Alto, CA) or 50\% v/v DS-A or $\mathrm{DS}+\mathrm{A}$. When present, the CD8+ T cell lines were assayed at $2 \times 10^{5} / \mathrm{ml}$. In some assays DS+A was preincubated for $30 \mathrm{~min}$ at $37^{\circ} \mathrm{C}$ with $100 \mathrm{NU} / \mathrm{ml}$ of polyclonal rabbit anti-human IL-12 (Genetics Institute) or $20 \mathrm{mg} / \mathrm{ml}$ monoclonal anti-IL-12 (Hoffmann-La Roche, Nutley, NJ) prior to addition to the PBMC. Supernatants were collected on day 4 , pooled, aliquotted and stored frozen prior to assay. $3 \mathrm{H}$-methyl thymidine $(1.0 \mathrm{mCi} / \mathrm{ml})$ was added to PBMC cultures on day 5 and the cells were harvested on day 6 . The small quantities of DS+A and DS-A available, as well as unavailability of long term cultured $\mathrm{T}$ cell lines, prevented measurement of the effects of DS+A, DS-A, rIL12, and anti-IL-10 on the growth kinetics of the T cell lines used in CD4S assays.

Maintenance of long term T cell cultures - Long term $\mathrm{P} 2$ polyclonal CD8+ T cell lines, $\mathrm{P} 2 \mathrm{~A} / 8 / 10 /$ 90 and P2A/8/1/91, polyclonal CD4+ T cell line,
$\mathrm{P} 2 \mathrm{~A} / 4 / 10 / 90$, and monoclonal CD4+ T cell line, $\mathrm{P} 2 \mathrm{~A} / 4 / 1$ were established from PBMC collected from P2 in 1990 during treatment for VL, as previously described (Holaday et al. 1993a). Similarly, P2 monoclonal CD8+ T cell lines, P2R/8/52 and $\mathrm{P} 2 \mathrm{R} / 8 / 53$ were established from PBMC collected from P2 after successful treatment. DTH+ adult Leishmania specific T1 CD4+ monoclonal T cell line, D4/46, and others used to generate CD4S (described below) were established from two DTH+ adults in 1990. All cell lines were stored frozen in liquid nitrogen. The T cells were thawed and expanded by stimulation with $5 \mathrm{mg} / \mathrm{ml}$ of PHA (Sigma Chemical Co, St. Louis, MO) in the presence of $25 \mathrm{U} / \mathrm{ml} \mathrm{rIL-2} \mathrm{(Cetus,} \mathrm{Emeryville,} \mathrm{CA),}$ $106 / \mathrm{ml}$ inactivated allogeneic PBMC and $10^{5} / \mathrm{ml}$ inactivated JY cells once per three weeks (Yssel et al. 1984). Allogeneic PBMC and JY were inactivated by exposure to $50 \mathrm{mg} / \mathrm{ml} \mathrm{mitomycin} \mathrm{c} \mathrm{for} 45$ min, three washes to remove the mitomycin c, and exposure to 2000 r g-irradiation.

IL-10 and IFN-gimmunoenzymetric ELISA - The levels of IL-10 in the supernatants were determined using commercially available monoclonal anti-human IL-10, anti-human IL-10-biotin, rhIL-10 (Pharmingen, San Diego, CA) and extravidin peroxidase (Sigma) according to Pharmingen's procedure. This ELISA is sensitive to $20 \mathrm{pg} / \mathrm{ml}$. IFN-g was measured using a commercially available human IFN-gELISA kit (Genzyme, Cambridge, MA). The kit is sensitive to at least $100 \mathrm{pg} / \mathrm{ml}$.

Production and assay of CD4S - Leishmania specific CD4+ T1 T cell clones isolated from two DTH+ adults and tested for Leishmania specificity, as previously described, were stimulated with PHA, allogeneic PBMC, JY and rIL-2 as described above (Holaday et al. 1993a). Supernatants were collected $72 \mathrm{hr}$ later, pooled and stored frozen prior to use. The levels of PHA and IL-2 remaining in CD4S were not mitogenic for PBMC. A small quantity of supernatant generated from the same CD4+ T cell clones by Leishmania antigen stimulation was also available for assay. The effects of CD4S on the kinetics of growth of CD4+ and CD8+ $\mathrm{T}$ cell lines was assessed by stimulating $\mathrm{T}$ cells with PHA in the presence or absence of 50\% v/v CD4S. To eliminate the possibility that the effects of CD4S on $\mathrm{T}$ cell lines might be enhanced by insufficient IL-2 the effects of doubling the concentration of IL-2 in CD4S assays was tested. Since DS+A is known to contain IFN-g, lymphotoxin and tumor necrosis factor-a, the effect of neutralizing antibodies specific for IFN-g $(30 \mathrm{mg} / \mathrm{ml}$, DNAX, Palo Alto, CA), lymphotoxin or tumor necrosis factora (4000 NU/ml), (Genentech, South San Francisco, CA) on CD4S activity was also determined. Half of the medium was removed twice per week and fresh medium, rIL-2, CD4S and antibody were 
added back to maintain the starting concentrations. After each week aliquots of the cells were counted and viability was assessed by trypan blue exclusion. Tests were carried out on a polyclonal CD4+ $\mathrm{T}$ cell line (P2A/4/10/90), a CD4+ clone derived from this line $(\mathrm{P} 2 \mathrm{~A} / 4 / 1)$, a CD4+ clone from a $\mathrm{DTH}+$ adult (D4/46), a polyclonal CD8+ $\mathrm{T}$ cell line derived from $\mathrm{P} 2$ during treatment $(\mathrm{P} 2 \mathrm{~A} / 8 / 10 /$ 90) and two CD8+ clones (P2R/8/52 and P2R/8/ 53) derived after $P 2$ had completed chemotherapy. CD4S assays were performed in the U.S. where PBMC from acute VL patients were unavailable and insufficient quantities of CD4S were available to measure the effects of CD4S on activities of acute VL patient PBMC.

Statistical analysis - The statistical significance of differences between means was determined by $t$ tests. A probability $<0.05$ was considered to be significant.

\section{RESULTS}

Assay 1 - Effects of immunomodulators on PBMC responses characteristic of VL

Existence of two categories of VL patients PBMC from VL patients could be placed in two groups, based on their level of endogenous IL-10 secretion, which responded differently to immunomodulators. PBMC displaying low levels
(7 of 13 assays) had an IL-10 secretion mean of 0.07 $\pm 0.06 \mathrm{ng} / \mathrm{ml}$ and $0 \pm 0 \mathrm{ng} / \mathrm{ml}$ of IFN-g (Table I). PBMC displaying high levels of endogenous IL-10 secretion (6 of 13 assays) had an IL-10 secretion mean of $0.71 \pm 0.21 \mathrm{ng} / \mathrm{ml}$ and $0 \pm 0.01 \mathrm{ng} / \mathrm{ml}$ of IFN-g(Table II). The probability of the high activity IL-10 values being sampled from the same population as the low activity group was only $8 \times 10^{-6}$.

PBMC from DTH+ adult controls had relatively low levels of endogenous IL-10 secretion (mean = $0.07 \pm 0.04 \mathrm{ng} / \mathrm{ml}), \mathrm{IFN}-g$ secretion $($ mean $=0 \pm 0$ ), and proliferation (mean $=3,876 \pm 1,904 \mathrm{cpm})$. Endogenous IL-10 and proliferation values were positively correlated $(\mathrm{r}=+0.83)$. The y axis intercept of the regression line was $0.0 \mathrm{ng} / \mathrm{ml}$ IL-10 (Fig. 1). DTH+ PBMC secreted $1.9 \pm 0.5 \mathrm{ng} / \mathrm{ml} \mathrm{IFN-g} \mathrm{and}$ $0.07 \pm 0.04 \mathrm{ng} / \mathrm{ml} \mathrm{IL}-10$ in response to antigen and had a mean proliferation SI of $11.8 \pm 4.5$.

Since levels of endogenous IL-10 secretion and proliferation were positively correlated in the DTH+ controls, PBMC from patients for whom only proliferation data were available were classified as having high or low activities by their level of endogenous proliferation. PBMC from patients with a mean endogenous $3 \mathrm{H}$-thymidine incorporation rate less than $3,650 \mathrm{cpm}$ (mean value for the low activity group +2 SD), P15, P18, P21, P26 and $\mathrm{P} 31$, were placed in the low activity group

TABLE I

Effects of Leishmania antigens and immunomodulators on IL-10 and IFN-gsecretion by peripheral blood mononuclear cells from low activity patients

\begin{tabular}{|c|c|c|c|c|c|c|c|c|}
\hline \multirow[b]{2}{*}{ Patient } & \multirow[b]{2}{*}{ Antigen } & \multicolumn{3}{|c|}{$\begin{array}{c}\mathrm{IL}-10 \\
\left(\mathrm{ng} / \mathrm{ml} \pm \mathrm{SD}^{a}\right)\end{array}$} & \multicolumn{4}{|c|}{$\begin{array}{c}\mathrm{IFN}-\mathrm{g} \\
(\mathrm{ng} / \mathrm{ml} \pm \mathrm{SD})\end{array}$} \\
\hline & & Med & $\mathrm{DS}+\mathrm{A}^{b}$ & IL-12 & Med & $\mathrm{DS}+\mathrm{A}$ & IL-12 & Anti-IL-10 \\
\hline \multirow[t]{2}{*}{24} & - & $.09 \pm .03$ & $.01 \pm .01$ & $.13 \pm .03$ & $0 \pm 0$ & $.06 \pm .01$ & $.18 \pm .04$ & $.21 \pm .01$ \\
\hline & + & $.19 \pm .01$ & $.27 \pm .12$ & $.07 \pm .02$ & $.08 \pm .10$ & $.06 \pm 0$ & $.16 \pm .02$ & $.24 \pm .01$ \\
\hline \multirow[t]{2}{*}{25} & - & $.16 \pm .0$ & $.18 \pm .01$ & $.15 \pm .06$ & $0 \pm 0$ & $.06 \pm .01$ & $.18 \pm .04$ & $.20 \pm .02$ \\
\hline & + & $.11 \pm .02$ & $.19 \pm .03$ & $.19 \pm .06$ & $.08 \pm .04$ & $.15 \pm .07$ & $.18 \pm .01$ & $.20 \pm .01$ \\
\hline \multirow[t]{2}{*}{27} & - & $.03 \pm 0$ & $.17 \pm .03$ & $.03 \pm 0$ & $0 \pm 0$ & $0 \pm 0$ & $0 \pm 0$ & $0 \pm 0$ \\
\hline & + & $.10 \pm 0$ & $.10 \pm .03$ & $.15 \pm .10$ & $0 \pm 0$ & $0 \pm 0$ & $0 \pm 0$ & $0 \pm 0$ \\
\hline \multirow[t]{2}{*}{28} & - & $.11 \pm .05$ & $.17 \pm .07$ & nd & $0 \pm 0$ & $.08 \pm .02$ & nd & nd \\
\hline & + & $.07 \pm .02$ & $.21 \pm .02$ & & $0 \pm 0$ & $.05 \pm 0$ & & \\
\hline \multirow[t]{2}{*}{29} & - & $.02 \pm .01$ & $.13 \pm .01$ & nd & $0 \pm 0$ & $.01 \pm .01$ & nd & nd \\
\hline & + & $.06 \pm .05$ & $.18 \pm 0$ & & $0 \pm 0$ & $.01 \pm 0$ & & \\
\hline \multirow[t]{2}{*}{30} & - & $0 \pm 0$ & $.18 \pm .06$ & $0 \pm 0$ & $0 \pm 0$ & $0 \pm 0$ & $.06 \pm .02$ & $.46 \pm 0$ \\
\hline & + & $0 \pm 0$ & $.18 \pm 0$ & $.02 \pm .02$ & $0 \pm 0$ & $0 \pm 0$ & $.06 \pm 0$ & $.72 \pm .34$ \\
\hline \multirow[t]{2}{*}{32} & - & $.05 \pm .01$ & $.19 \pm .02$ & nd & $0 \pm 0$ & $.04 \pm .06$ & nd & nd \\
\hline & + & $.11 \pm .01$ & $.19 \pm .01$ & & $0 \pm 0$ & $.08 \pm 0$ & & \\
\hline \multirow[t]{2}{*}{ Mean } & - & $.07 \pm .06$ & $.15 \pm .06$ & $.08 \pm .07$ & $0 \pm 0$ & $.04 \pm .03$ & $.10 \pm .09$ & $.22 \pm .19$ \\
\hline & + & $.09 \pm .06$ & $.19 \pm .05$ & $.11 \pm .08$ & $.02 \pm .04$ & $.05 \pm .05$ & $.10 \pm .08$ & $.29 \pm .30$ \\
\hline
\end{tabular}

$a$ : mean $\mathrm{ng} / \mathrm{ml} \pm 1$ standard deviation; $b$ : supernatant derived by stimulation of peripheral blood mononuclear cells from skin test positive adults with Leishmania antigens. 
TABLE II

Effects of Leishmania antigens and immunomodulators on IL-10 and IFN-gsecretion by peripheral blood mononuclear cells from high activity patients

\begin{tabular}{|c|c|c|c|c|c|c|c|c|}
\hline \multirow[b]{2}{*}{ Patient } & \multirow[b]{2}{*}{ Antigen } & \multicolumn{3}{|c|}{$\begin{array}{c}\mathrm{IL}-10 \\
\left(\mathrm{ng} / \mathrm{ml} \pm \mathrm{SD}^{a}\right)\end{array}$} & \multicolumn{4}{|c|}{$\begin{array}{c}\mathrm{IFN}-\mathrm{g} \\
(\mathrm{ng} / \mathrm{ml} \pm \mathrm{SD})\end{array}$} \\
\hline & & Med & $\mathrm{DS}+\mathrm{A}^{b}$ & IL-12 & Med & $\mathrm{DS}+\mathrm{A}$ & IL-12 & Anti-IL-10 \\
\hline \multirow[t]{2}{*}{17} & - & $.46 \pm .03$ & $.13 \pm .01$ & $.37 \pm .04$ & $0 \pm 0$ & $.09 \pm .01$ & $.23 \pm 0$ & nd \\
\hline & + & $.11 \pm .01$ & $.10 \pm .02$ & $.33 \pm .06$ & $.03 \pm 0$ & $.12 \pm .01$ & $.21 \pm .01$ & \\
\hline \multirow[t]{2}{*}{19} & - & $.84 \pm .01$ & $.17 \pm .01$ & $.73 \pm .01$ & $0 \pm 0$ & $.04 \pm .05$ & $.23 \pm 0$ & $.05 \pm .01$ \\
\hline & + & $.19 \pm .02$ & $.11 \pm .03$ & $.34 \pm .01$ & $.11 \pm .11$ & $.10 \pm .04$ & $.21 \pm 0$ & $.16 \pm .06$ \\
\hline \multirow[t]{2}{*}{$19 \mathrm{R}$} & - & $.99 \pm .19$ & $.29 \pm .02$ & $.94 \pm .05$ & $.02 \pm .04$ & $.08 \pm .02$ & $.10 \pm .14$ & $.06 \pm .04$ \\
\hline & + & $.18 \pm .01$ & $.20 \pm .01$ & $.42 \pm .02$ & $0 \pm 0$ & $.09 \pm .01$ & $0 \pm 0$ & $.10 \pm .04$ \\
\hline \multirow[t]{2}{*}{20} & - & $.67 \pm .01$ & $0 \pm 0$ & $.70 \pm .02$ & $0 \pm 0$ & $.13 \pm 0$ & $.02 \pm .02$ & nd \\
\hline & + & $.14 \pm .16$ & $.08 \pm .07$ & $.44 \pm .21$ & $.18 \pm 0$ & $.15 \pm 0$ & $.24 \pm .04$ & \\
\hline \multirow[t]{2}{*}{22} & - & $.80 \pm .11$ & $.54 \pm .11$ & $.83 \pm .03$ & $0 \pm 0$ & $.10 \pm 0$ & $.26 \pm .06$ & $.05 \pm .07$ \\
\hline & + & $.25 \pm .03$ & $.28 \pm .02$ & $.74 \pm .08$ & $.12 \pm .02$ & $.12 \pm .02$ & $.25 \pm 0$ & $.18 \pm .11$ \\
\hline \multirow[t]{2}{*}{23} & - & $.49 \pm .08$ & $.43 \pm .13$ & $.97 \pm .16$ & $0 \pm 0$ & $.12 \pm .06$ & $.04 \pm .01$ & $0 \pm 0$ \\
\hline & + & $1.08 \pm .03$ & $.65 \pm .06$ & $.80 \pm .10$ & $.08 \pm 0$ & $.14 \pm .02$ & $.28 \pm 0$ & $.10 \pm .01$ \\
\hline \multirow[t]{2}{*}{ Mean } & - & $.71 \pm .21$ & $.26 \pm .20$ & $.76 \pm .22$ & $0 \pm .01$ & $.09 \pm .03$ & $.15 \pm .11$ & $.04 \pm .03$ \\
\hline & + & $.32 \pm .37$ & $.24 \pm .22$ & $.51 \pm .20$ & $.09 \pm .06$ & $.12 \pm .02$ & $.20 \pm .10$ & $.14 \pm .04$ \\
\hline
\end{tabular}

$a$ : mean $\mathrm{ng}$ per $\mathrm{ml} \pm 1$ standard deviation; $b$ : supernatant derived by stimulation of peripheral blood mononuclear cells from skin test positive adults with Leishmania antigens.

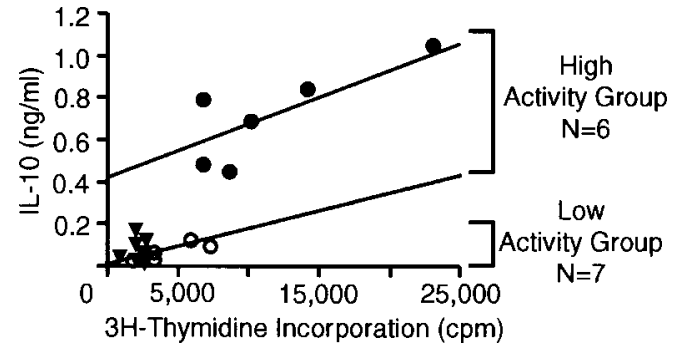

Fig. 1: relationship between endogenous IL-10 secretion and proliferation by peripheral blood mononuclear cells (PBMC) from DTH+ adults (hollow circles), low activity visceral leishmaniasis patients (VL) (triangles), and high activity VL patients (solid circles). Regression lines for DTH+ adult and high activity VL patient PBMC are also shown.

(Table III). PBMC from patients with a mean endogenous $3 \mathrm{H}$-thymidine incorporation rate greater than $5,225 \mathrm{cpm}$ (mean value for the low activity group +4 SD), P14 and P16, were placed in the high activity group (Table IV). PBMC from the low activity group had a proliferation mean of $2,073 \pm 788 \mathrm{cpm}$. These values were not correlated with endogenous IL-10 secretion $(r=+0.15)$ but both variables were low compared to high activity group levels. PBMC in this group had a mean proliferation SI of $1.8 \pm 1.1$ (not including P18 PBMC which had an atypically high SI of 24). The endogenous proliferation mean for the high activ- ity group was $12,922 \pm 7,651$; levels of endogenous IL-10 secretion and proliferation were correlated for this group $(r=+0.76)$. The y axis intercept of the regression line relating the two variables was 0.42 ng/ml IL-10 (Fig. 1).

Effects of immunomodulators on cytokine secretion by VL patient PBMC- The effects of Leishmania antigens, by themselves or as part of DS+A, on IL-10 secretion by acute VL patient PBMC were different in assays of high and low activity patients. PBMC in the low activity group did not show significant changes in IL-10 or IFN-gsecretion in response to antigen alone. DS+A produced a small but significant increase in IL-10 secretion. IL-12 and anti-IL-10 caused small but significant increases in IFN-gsecretion with or without antigen present. In contrast, PBMC in the high activity group overall secreted less IL-10 in response to antigen. DS+A caused changes similar to antigen alone. IL-12 had no consistent effect on IL-10 secretion except to decrease the effect of antigen on secretion. Antigen alone or in DS+A caused a very small but significant increase in IFN-g secretion. IL-12 caused a significant increase in IFN-gsecretion with or without antigen present. Anti-IL-10 had no significant effect on IFN-g secretion.

Effects of immunomodulators on proliferation by VL patient $P B M C$ - The immunomodulators also had different effects on proliferation in assays of PBMC with high and low endogenous activities. 


\section{TABLE III}

Effects of Leishmania antigens and immunomodulators on proliferation by peripheral blood mononuclear cells from low activity patients

\begin{tabular}{|c|c|c|c|c|c|c|c|c|}
\hline Patients & Antigen & Medium & $\mathrm{DS}+\mathrm{A}^{a}$ & $\begin{array}{c}\mathrm{SI}^{b} \\
\mathrm{DS}+\mathrm{A}\end{array}$ & IL-12 & $\begin{array}{c}\text { SI } \\
\text { IL-12 }\end{array}$ & Anti-IL-10 & $\begin{array}{c}\text { SI } \\
\text { anti-IL-10 }\end{array}$ \\
\hline 15 & $\stackrel{-}{+}$ & $\begin{array}{c}1,038 \pm 64^{c} \\
2,401 \pm 151 \\
2.3\end{array}$ & $\begin{array}{c}2,731 \pm 595 \\
3,884 \pm 476 \\
1.4\end{array}$ & 2.6 & $\begin{array}{c}1,075 \pm 269 \\
3,573 \pm 180 \\
3.3\end{array}$ & 1.0 & nd & \\
\hline 18 & $\stackrel{-}{+}$ & $\begin{array}{c}1,406 \pm 202 \\
33,384 \pm 2,464 \\
24\end{array}$ & $\begin{array}{c}13,771 \pm 634 \\
46,364 \pm 2,331 \\
3.4\end{array}$ & 9.8 & nd & & nd & \\
\hline 21 & $\begin{array}{c}- \\
+ \\
\text { SI Antigen }\end{array}$ & $\begin{array}{c}2,518 \pm 414 \\
5,254 \pm 692 \\
2.1\end{array}$ & $\begin{array}{c}8,489 \pm 1,411 \\
8,646 \pm 1,034 \\
1.0\end{array}$ & 3.4 & nd & & $\begin{array}{c}4,968 \pm 820 \\
7,686 \pm 364 \\
1.6\end{array}$ & 2.0 \\
\hline 24 & $\begin{array}{c}- \\
+ \\
\text { SI Antigen }\end{array}$ & $\begin{array}{c}2,160 \pm 246 \\
2,045 \pm 534 \\
1.0\end{array}$ & $\begin{array}{c}2,655 \pm 599 \\
4,703 \pm 1,810 \\
1.8\end{array}$ & 1.2 & $\begin{array}{c}7,257 \pm 2,388 \\
6,290 \pm 365 \\
0.9\end{array}$ & 3.4 & $\begin{array}{c}2,278 \pm 488 \\
5,341 \pm 656 \\
2.3\end{array}$ & 1.0 \\
\hline 25 & $\stackrel{-}{+}+$ & $\begin{array}{c}2,183 \pm 83 \\
1,705 \pm 132 \\
0.8\end{array}$ & $\begin{array}{c}2,577 \pm 463 \\
3,511 \pm 1,728 \\
1.4\end{array}$ & 1.2 & $\begin{array}{c}6,228 \pm 1,600 \\
5,414 \pm 986 \\
0.9\end{array}$ & 2.8 & $\begin{array}{c}4,114 \pm 1,228 \\
2,505 \pm 600 \\
0.6\end{array}$ & 1.9 \\
\hline 26 & $\stackrel{-}{+}$ & $\begin{array}{c}3,225 \pm 3,087 \\
2,614 \pm 326 \\
0.8\end{array}$ & $\begin{array}{c}8,038 \pm 524 \\
8,164 \pm 1,503 \\
1.0\end{array}$ & 2.5 & nd & & nd & \\
\hline 27 & $\stackrel{-}{+}$ & $\begin{array}{c}983 \pm 60 \\
1,179 \pm 400 \\
1.2\end{array}$ & $\begin{array}{c}1,998 \pm 731 \\
4,369 \pm 488 \\
2.2\end{array}$ & 2.0 & $\begin{array}{c}3,374 \pm 650 \\
3,644 \pm 880 \\
1.1\end{array}$ & 3.4 & $\begin{array}{c}7,396 \pm 693 \\
6,839 \pm 4,540 \\
0.9\end{array}$ & 7.5 \\
\hline 28 & $\stackrel{-}{+}+$ & $\begin{array}{c}2,889 \pm 1,700 \\
9,922 \pm 1,644 \\
3.4\end{array}$ & $\begin{array}{c}11,996 \pm 1,112 \\
18,782 \pm 4,522 \\
1.6\end{array}$ & 4.2 & $\begin{array}{c}7,820 \pm 2,084 \\
21,621 \pm 3,609 \\
2.8\end{array}$ & 2.8 & nd & \\
\hline 29 & $\stackrel{-}{+}$ & $\begin{array}{c}2,136 \pm 79 \\
2,290 \pm 493 \\
1.1\end{array}$ & $\begin{array}{c}3,851 \pm 632 \\
8,330 \pm 371 \\
2.2\end{array}$ & 1.8 & $\begin{array}{c}2,927 \pm 370 \\
9,893 \pm 573 \\
3.4\end{array}$ & 1.4 & nd & \\
\hline 30 & $\stackrel{-}{+}+$ & $\begin{array}{c}2,722 \pm 1,086 \\
3,978 \pm 2,001 \\
1.5\end{array}$ & $\begin{array}{c}5,069 \pm 486 \\
11,432 \pm 2,248 \\
2.2\end{array}$ & 1.9 & $\begin{array}{c}4,537 \pm 2,007 \\
6,050 \pm 726 \\
1.3\end{array}$ & 1.7 & $\begin{array}{c}5,852 \pm 1,719 \\
1,651 \pm 319 \\
0.3\end{array}$ & 2.1 \\
\hline 31 & $\stackrel{-}{+}+$ & $\begin{array}{c}988 \pm 356 \\
1,202 \pm 211 \\
1.2\end{array}$ & $\begin{array}{c}1,888 \pm 375 \\
2,895 \pm 565 \\
1.5\end{array}$ & 1.9 & $\begin{array}{c}878 \pm 372 \\
1,819 \pm 444 \\
2.1\end{array}$ & 0.9 & nd & \\
\hline 32 & $\stackrel{-}{+}$ & $\begin{array}{c}2,627 \pm 614 \\
10,967 \pm 1,918 \\
4.2\end{array}$ & $\begin{array}{c}8,513 \pm 742 \\
12,203 \pm 1,209 \\
1.4\end{array}$ & 3.2 & $\begin{array}{c}6,028 \pm 936 \\
18,038 \pm 2,447 \\
3.0\end{array}$ & 2.3 & nd & \\
\hline $\begin{array}{l}\text { Mean SI } \\
\text { values }^{d}\end{array}$ & & $\begin{array}{l}1.8 \pm 1.1 \\
\text { antigen } \\
\text { only }\end{array}$ & $\begin{array}{c}1.6 \pm 0.4 \\
\text { antigen }+ \\
\mathrm{DS}+\mathrm{A}\end{array}$ & $\begin{array}{c}2.4 \pm 0.9 \\
\mathrm{DS}+\mathrm{A} \\
\text { only }\end{array}$ & $\begin{array}{l}2.1 \pm 1.1 \\
\text { antigen } \\
+\mathrm{IL}-12\end{array}$ & $\begin{array}{c}2.2 \pm 1.0 \\
\text { IL-12 } \\
\text { only }\end{array}$ & $\begin{array}{c}1.1 \pm 0.8 \\
\text { antigen } \\
+ \text { anti-IL-10 }\end{array}$ & $\begin{array}{r}2.9 \pm 2.6 \\
\text { anti-IL-10 } \\
\text { only }\end{array}$ \\
\hline
\end{tabular}

$a$ : supernatant derived by stimulation of peripheral blood mononuclear cells from skin test positive adults with Leishmania antigens; $b$ : stimulation index; $c$ : mean counts per minute \pm 1 standard deviation; $d$ : do not include P18 data which were atypical for visceral leishmaniasis patients.

None of the immunomodulators had a significant effect on proliferation in the low activity assays (Table III). DS+A alone decreased endogenous proliferation in assays of high activity PBMC producing a mean SI of $0.7 \pm 0.3$, significantly less than the SI for antigen alone, $1.4 \pm 0.8(\mathrm{p}=0.04)$, or antigen plus DS+A, $2.3 \pm 0.8,(\mathrm{p}=0.0001)$, (Table IV). Anti-IL-10 significantly increased proliferation in response to antigen $(\mathrm{p}=0.04)$. Pretreatment of DS+A with $100 \mathrm{NU} / \mathrm{ml}$ polyclonal or $20 \mathrm{mg} / \mathrm{ml}$ 
TABLE IV

Effects of Leishmania antigens and immunomodulators on proliferation by peripheral blood mononuclear cells from high activity visceral leishmaniasis patients

\begin{tabular}{|c|c|c|c|c|c|c|c|c|}
\hline Patients & s Antigen & Medium & $\mathrm{DS}+\mathrm{A}^{a}$ & $\begin{array}{c}\mathrm{SI}^{b} \\
\mathrm{DS}+\mathrm{A}\end{array}$ & IL-12 & $\begin{array}{c}\text { SI } \\
\text { IL-12 }\end{array}$ & Anti-IL-10 & $\begin{array}{c}\text { SI } \\
\text { anti-IL-10 }\end{array}$ \\
\hline 14 & $\begin{array}{c}- \\
+ \\
\text { SI Antigen }\end{array}$ & $\begin{array}{c}26,152 \pm 4,786^{\mathrm{c}} \\
17,044 \pm 3,305 \\
0.6\end{array}$ & $\begin{array}{c}7,557 \pm 1,675 \\
15,066 \pm 925 \\
2.0\end{array}$ & 0.3 & $\begin{array}{c}32,320 \pm 4,412 \\
32,585 \pm 5,174 \\
1.0\end{array}$ & 1.2 & nd & \\
\hline 16 & $\begin{array}{c}- \\
+ \\
\text { SI Antigen }\end{array}$ & $\begin{array}{c}7,819 \pm 1,103 \\
13,505 \pm 1,178 \\
1.7\end{array}$ & $\begin{array}{c}7,412 \pm 1,443 \\
17,086 \pm 2,184 \\
2.3\end{array}$ & 1.0 & $\begin{array}{c}23,464 \pm 1,914 \\
35,225 \pm 5,509 \\
1.5\end{array}$ & 3.0 & nd & \\
\hline 17 & $\begin{array}{c}- \\
+ \\
\text { SI Antigen }\end{array}$ & $\begin{array}{c}8,582 \pm 1,162 \\
7,161 \pm 2,497 \\
0.8\end{array}$ & $\begin{array}{c}6,890 \pm 1,527 \\
7,266 \pm 431 \\
1.0\end{array}$ & 0.8 & $\begin{array}{c}12,075 \pm 2,862 \\
9,664 \pm 368 \\
0.8\end{array}$ & 1.4 & nd & \\
\hline 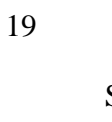 & $\begin{array}{c}- \\
+ \\
\text { SI Antigen }\end{array}$ & $\begin{array}{c}14,145 \pm 4,513 \\
18,118 \pm 3,146 \\
1.3\end{array}$ & $\begin{array}{c}7,035 \pm 728 \\
26,234 \pm 507 \\
3.7\end{array}$ & 0.5 & $\begin{array}{c}23,456 \pm 1,620 \\
49,461 \pm 95 \\
2.1\end{array}$ & 1.7 & $\begin{array}{c}20,536 \pm 790 \\
43,320 \pm 911 \\
2.1\end{array}$ & 1.4 \\
\hline $19 \mathrm{R}$ & $\begin{array}{c}- \\
+ \\
\text { SI Antigen }\end{array}$ & $\begin{array}{c}23,099 \pm 3,424 \\
42,178 \pm 5,432 \\
1.8\end{array}$ & $\begin{array}{c}17,724 \pm 3,218 \\
55,418 \pm 9,457 \\
3.1\end{array}$ & 0.8 & $\begin{array}{c}45,065 \pm 1,858 \\
110,001 \pm 4,684 \\
2.4\end{array}$ & 2.0 & $\begin{array}{c}46,019 \pm 3,822 \\
98,001 \pm 7,530 \\
2.1\end{array}$ & 2.0 \\
\hline 20 & $\begin{array}{c}- \\
+ \\
\text { SI Antigen }\end{array}$ & $\begin{array}{c}10,163 \pm 3,337 \\
9,311 \pm 3,137 \\
0.9\end{array}$ & $\begin{array}{c}7,444 \pm 1,665 \\
11,551 \pm 2,495 \\
1.6\end{array}$ & 0.7 & $\begin{array}{c}15,331 \pm 1,800 \\
22,061 \pm 3,954 \\
1.4\end{array}$ & 1.5 & nd & \\
\hline 22 & $\begin{array}{c}- \\
+ \\
\text { SI Antigen }\end{array}$ & $\begin{array}{c}6,686 \pm 2,688 \\
19,579 \pm 2,934 \\
2.9\end{array}$ & $\begin{array}{c}8,567 \pm 3,036 \\
20,067 \pm 4,349 \\
2.3\end{array}$ & 1.3 & $\begin{array}{c}10,776 \pm 1,629 \\
43,257 \pm 3,412 \\
4.0\end{array}$ & 1.6 & $\begin{array}{c}8,600 \pm 5,152 \\
34,420 \pm 3,907 \\
4.0\end{array}$ & 1.3 \\
\hline 23 & $\begin{array}{c}- \\
+ \\
\text { SI Antigen }\end{array}$ & $\begin{array}{c}6,731 \pm 2,364 \\
7,021 \pm 678 \\
1.0\end{array}$ & $\begin{array}{c}2,638 \pm 642 \\
5,651 \pm 793 \\
2.1\end{array}$ & 0.4 & $\begin{array}{c}5,250 \pm 1,170 \\
6,737 \pm 1,110 \\
1.3\end{array}$ & 0.8 & $\begin{array}{c}8,670 \pm 2,722 \\
16,851 \pm 2,167 \\
1.9\end{array}$ & 1.3 \\
\hline $\begin{array}{l}\text { Mean SI } \\
\text { values }\end{array}$ & & $\begin{array}{c}1.4 \pm 0.8 \\
\text { antigen } \\
\text { only }\end{array}$ & $\begin{array}{l}2.3 \pm 0.8 \\
\text { antigen } \\
+\mathrm{DS}+\mathrm{A}\end{array}$ & $\begin{array}{c}0.7 \pm 0.3 \\
\text { DS }+ \text { A } \\
\text { only }\end{array}$ & $\begin{array}{l}1.8 \pm 1.0 \\
\text { antigen } \\
+ \text { IL-12 }\end{array}$ & $\begin{array}{l}1.6 \pm 0.6 \\
\text { IL-12 } \\
\text { only }\end{array}$ & $\begin{array}{c}2.5 \pm 1.0 \\
\text { antigen } \\
+ \text { anti-IL-10 }\end{array}$ & $\begin{array}{c}1.5 \pm 0.3 \\
\text { IL-10 } \\
\text { only }\end{array}$ \\
\hline
\end{tabular}

$a$ : supernatant derived by stimulation of peripheral blood mononuclear cells from skin test positive adults with Leishmania antigens; $b$ : stimulation index $c$ : mean counts per minute \pm 1 standard deviation.

monoclonal anti-IL-12 did not reverse the effects of DS+A (data not shown). IL-12 alone did not have a consistent effect on proliferation. Stimulation of $\mathrm{DTH}+$ adult control PBMC with DS+A increased endogenous proliferation $(\mathrm{SI}=11.5 \pm 3.1)$ by the same amount as antigen only (11.8 \pm 4.5$)$.

Assay 2 - Ability of immunomodulators to inhibit $C D 8+T s$ mediated changes in cytokine secretion and proliferation - The addition of CD8+ $\mathrm{T}$ cells isolated during acute phase VL to autologous PBMC collected after successful treatment has been shown to result in increased IL-10 secretion in the absence or presence of added Leishmania antigens (Holaday et al. 1993a). The ability of DS+A and IL-12 to reverse this increase was tested (Table V). PBMC collected from a VL patient (P2) three years after successful treatment secreted 0.10 $\pm 0.08 \mathrm{ng} / \mathrm{ml} \mathrm{IL}-10$ in the absence of antigen and
$0.49 \pm 0 \mathrm{ng} / \mathrm{ml}$ in the presence of antigen. Addition of the autologous CD8+ T cell line, P2A/8/1/ 91 to these PBMC increased IL-10 secretion to 1.42 $\pm 0.07 \mathrm{ng} / \mathrm{ml}$ in the absence of antigen. Addition of antigen decreased this high level two-fold, similar to the effect of antigen on endogenous IL-10 secretion by PBMC from high activity patients. Addition of DS+A alone increased PBMC IL-10 secretion by the same amount as antigen alone but prevented IL-10 secretion from increasing in response to the addition of CD8+ T cells twice as well as antigen alone. IL-12 increased IL-10 secretion in the absence of added antigen by about the same amount as the CD8+ T cells. The PBMC did not secrete IFN-gin any of the assays. CD8+ $\mathrm{T}$ cells inhibited antigen specific proliferation by about $50 \%$. DS+A, but not IL-12, prevented the decrease (data not shown). 
TABLE V

Effects of Leishmania antigens, DS+A, and IL-12 on IL-10 secretion by an acute phase CD8+ T cell line mixed with autologous peripheral blood mononuclear cells from a recovered visceral leishmaniasis patient

\begin{tabular}{ccccc}
\hline & & \multicolumn{3}{c}{$\begin{array}{c}\text { IL-10 } \\
{\left[\mathrm{ng} / \mathrm{ml}\left(\mathrm{mean} \pm \mathrm{SD}^{a}\right)\right]} \\
\text { Immunomodulators added }\end{array}$} \\
\cline { 3 - 5 } $\begin{array}{l}\text { CD8+ T } \\
\text { cells added }\end{array}$ & Antigen & Medium & $\begin{array}{c}\mathrm{DS}+\mathrm{A}^{b} \\
(50 \% \mathrm{v} / \mathrm{v})\end{array}$ & $\begin{array}{c}\mathrm{IL}-12 \\
(50 \mathrm{U} / \mathrm{ml})\end{array}$ \\
\hline- & - & $0.10 \pm 0.08$ & $0.48 \pm 0.06$ & $1.28 \pm 0.04$ \\
+ & + & $0.49 \pm 0$ & $0.33 \pm 0.10$ & $0.77 \pm 0$ \\
& - & $1.42 \pm 0.07$ & $0.40 \pm 0.01$ & $0.85 \pm 0.01$ \\
\hline
\end{tabular}

$a$ : standard deviation $b$ : supernatant derived by stimulation of peripheral blood mononuclear cells from skin test positive adults with Leishmania antigens.

Assay 3 - The effects of CD4S on the growth of $C D 4+$ and $C D 8+T$ cell lines - Pooled supernatant from Leishmania specific T1 CD4+ T cells (CD4S) had a differential effect on the growth of CD4+ and CD8+ T cell lines (Fig. 2). CD4S did not affect the growth of CD8+ cells during the first week in culture, but CD8+ cells began to die during the second week of culture with CD4S and nearly all of them were dead by four weeks. Doubling the concentration of rIL-2 or adding neutralizing antibodies to INF-g, lymphotoxin or tumor necrosis factor-a did not abrogate this effect. In fact, extra rIL-2 inhibited CD8+ T cell proliferation during the first week of culture (Fig. 3).
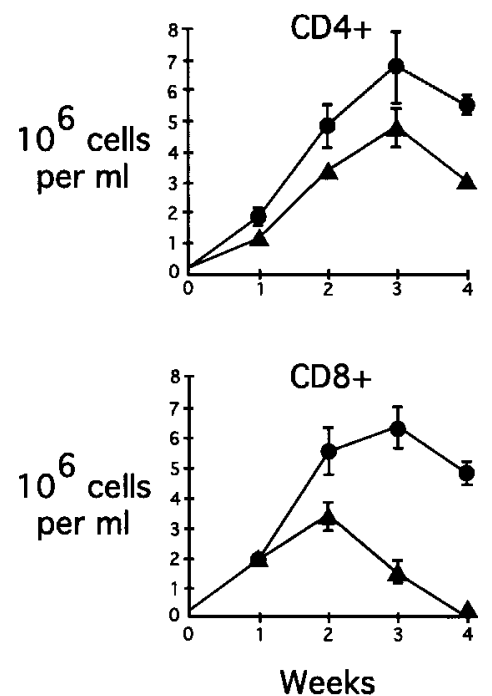

Fig. 2: growth of CD4+ T cells (D4/46) and CD8+ T cells ( $\mathrm{P} 2 \mathrm{R} / 8 / 53)$ in the presence (triangles) or absence (circles) of CD4S (supernatant generated by PHA stimulation of pooled Leishmania specific T cell clones). Each data point represents the mean \pm the standard deviation of a triplicate assay.
CD4S partially inhibited the growth of CD4+ $\mathrm{T}$ cell lines but did not result in cell death. The effect was completely abrogated by doubling the concentration of rIL-2 in the media to a final concentration of $50 \mathrm{U} / \mathrm{ml}$ (Fig. 3). Experiments with a supernatant derived by antigen-stimulation of CD4+ T cell clones from the same two DTH+ adults produced similar changes in growth kinetics (data not shown).

\section{DISCUSSION}

An unexpected finding of this study was the discovery of two categories of VL patients, those with high and low levels of endogenous IL-10 secretion and proliferation, which responded differently to immunomodulators. These groups may correspond to groups of patients with high and low levels of serum IL-4 (Zwingenberger et al. 1990, Holaday et al. 1993b). The existence of groups of VL patients with high and low levels of endogenous IL-10 secretion has not been reported by other researchers who assayed PBMC at lower cell densities in less enriched media and at lower serum concentrations (Ghalib et al. 1993, 1995, Carvalho et al. 1994). Ghalib et al. (1995) assayed PBMC which had been frozen prior to culturing so that any PBMC activities occurring when the cells were first isolated may have been terminated before the assays were performed. Patients in this study were adults $(14-58$, mean $=28$ years $)$ living in both endemic and epidemic areas, rather than only adults in epidemic areas or small children. As explained below, subjects without previous exposure to Leishmania might rarely produce immune responses like those in the high endogenous activity group. High endogenous activity PBMC patients have been observed by members of the Núcleo de Medicina Tropical since 1989 using different lots of sera. The most stringent conditions 
$1 \times \mathrm{IL}-2$
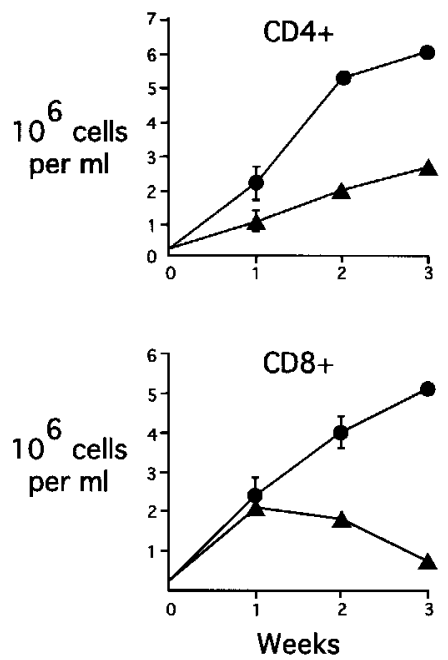

$2 X \mathrm{XL}-2$
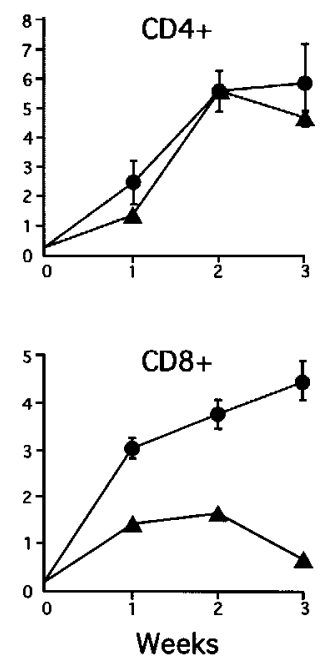

Fig. 3: growth of CD4+ T cells ( $\mathrm{P} 2 \mathrm{~A} / 4 / 10 / 90)$ and $\mathrm{CD} 8+\mathrm{T}$ cells $(\mathrm{P} 2 \mathrm{R} / 8 / 10 / 90)$ in the presence (triangles) or absence (circles) of CD4S (supernatant generated by PHA stimulation of pooled Leishmania specific T cell clones) and the effect of $25 \mathrm{U} / \mathrm{ml} \mathrm{rIL}-2$ $(1 \mathrm{X})$ or $50 \mathrm{U} / \mathrm{ml} \mathrm{rIL-2}(2 \mathrm{X})$ on CD4S inhibition of CD4+ and CD8+ T cell growth. Each data point represents the mean \pm the standard deviation of five assays.

were used in the assays reported here to prevent endotoxin contamination as described in Materials and Methods and the same sera were used in all the assays. In addition, high endogenous activities were not observed in any of ten assays of PBMC from DTH+ adults which were performed using the same media. Thus, it is safe to assume that the high endogenous levels were not the result of endotoxin contamination or differences in sera.

Elevated endogenous values could be explained in part by Leishmania independent immune responses because poor, rural inhabitants of northeastern Brazil are usually infected with intestinal helminths and other pathogens but the highest values in this study were unique to VL patient PBMC. The high values may be correlated to disease progression, similar to the positive correlation between level of endogenous IL-10 secretion by PBMC and alveolar macrophages from HIV+ subjects and CDC staging (Barcellini et al. 1994, Denis \& Ghadirian 1994). Criteria for comparable staging of VL patients are not available but the fact that P14 and P23, who had been symptomatic the longest and were not responding well to therapy, were among the high value patients supports this theory.

Alternatively, more than one type of immunopathology may lead to delevopment of VL, similar to the differences in immunopathology associated with lepromatous and tuberculoid forms of leprosy (Salgame et al. 1991). For example, people who have never been exposed to L. chagasi, such as those infected during epidemics, might develop clinical symptoms because they failed to develop protective immunity fast enough. All seven of the patients who lived in areas experiencing epidemic outbreaks (P18, P21, P26, P27, P29, P30, and P31) were in the low activity group. Adults who develop clinical symptoms of VL only after years of residence in an endemic area and those who have recovered from VL but relapse or are reinfected presumably already have protective immunity but may have developed symptomatic infections when their immunity was compromised by concurrent infections, allergies, malnutrition or other causes. There is evidence to support this theory. P2 had $\mathrm{T} 1$ or mixed T1/T2 responses to Leishmania antigens in assays performed one year after successful treatment for VL but had $\mathrm{T} 2$ responses to the antigens three years after treatment (Table V) (Holaday et al. 1993a). The T2 response occurred during a time when P2 suffered from undiagnosed gastrointestinal symptoms of more than two months duration. P2 also had some asthma symptoms during this time. A DTH+ adult, D4, who had a strong T1 CD4+ T cell response to Leishmania antigens in 1990 produced PBMC which had a high endogenous level of IL-10 secretion $(0.41 \pm 0 \mathrm{ng} / \mathrm{ml})$ in 1992. Stimulation of the PBMC with Leishmania antigens reduced IL- 10 secretion $(0 \pm 0 \mathrm{ng} / \mathrm{ml})$ but failed to cause a measureable increase in IFN-g 
secretion. This atypical DTH+ adult response was similar to those of acute patients in the high activity group and occurred at a time when the subject had been ill of undiagnosed causes for at least one month. If P2 or D4 had been infected with $L$. chagasi at the time they had $\mathrm{T} 2$ responses they might have developed symptomatic infections. The occurence of $\mathrm{T} 2$ responses in a treated patient and a DTH+ adult during illness suggests that immune responses to $L$. chagasi vary along a continuum when the immune system responds to changing demands.

Several lines of evidence suggest that an increased frequency or activity of a PBMC subset is responsible for the high levels of endogenous IL10 secretion, and by correlation, proliferation also. A comparison of the $y$ axis intercept values of high activity VL patients $(0.42 \mathrm{ng} / \mathrm{ml} \mathrm{IL}-10)$ and DTH+ adult controls $(0.0 \mathrm{ng} / \mathrm{ml} \mathrm{IL-10)})$ demonstrates that high activity patient PBMC must have had an increased frequency of a PBMC subset or a subset in a different activation state than the DTH+ adult controls. Acute phase CD8+ T cells, but not CD4+ $\mathrm{T}$ cells, have been shown to mediate increased IL10 secretion when mixed with autologous PBMC collected after successful treatment of VL in the presence or absence of added antigen (Table V), (Holaday et al. 1993a). In addition, preliminary experiments of CD8+ T cell depletion of VL patient PBMC have shown that depletion of CD8+ T cells ablates endogenous IL-10 secretion but not antigen specific IL-10 secretion (Holaday, unpublished observations). An increased frequency of T2 CD8+ T cells has also been observed in some CDC stage IV AIDS patients (Maggi et al. 1994).

Assays of the effects of immunomodulators on the activities of unfractionated PBMC detected two activities in DS+A which inhibited high endogenous activities of PBMC from VL patients. Leishmania antigens, by themselves or as part of DS+A, decreased high endogenous IL-10 secretion by VL patient PBMC. Similarly the antigens by themselves or as part of DS+A prevented the CD8+ T cell mediated IL-10 secretion increase from occurring in the $\mathrm{P} 2$ reconstitution assay. The ability of Leishmania antigens to lower IL-10 secretion is intriguing in light of reports of successful immunotherapy of cutaneous leishmaniasis with Leishmania antigens plus BCG (Convit et al. 1987, 1989). The means by which Leishmania antigens decreased IL-10 secretion are not known. IL-12 did not decrease IL-10 secretion and reduced the inhibitory effects of antigen on IL-10 secretion. INF-ghas been shown to decrease IL-10 secretion by monocytes and PBMC (Chomarat et al. 1993). However, PBMC secretion of IFN-g in response to antigen stimulation does not appear to be re- sponsible for the Leishmania antigens' effect on IL-10 secretion because PBMC secretion of IFN-g and IL-10 in response to antigen were not correlated in the high activity group of patients $(\mathrm{r}=$ +.06 ). Similarly, Karp et al found that levels of IL-10 and IFN-g mRNA in bone marrow of VL patients were not inversely correlated (Karp et al. 1993).

A second DS+A activity decreased high endogenous proliferation without decreasing antigen specific responses, effectively increasing antigen specific proliferation. The effect of DS+A on proliferation cannot be explained by a "spent medium effect" because DS+A decreased endogenous proliferation only in assays of high activity VL patient PBMC. It increased endogenous proliferation in assays of low activity VL patient and DTH+ adult control PBMC. The identity of the factor(s) responsible for DS+A activity is not yet known but does not appear to include IL-12 or cytokines which act by reducing IL-10 secretion. If high endogenous proliferation was mediated by $\mathrm{T} 2$ CD4+ $\mathrm{T}$ cells the IFN-g present in DS+A might explain the ability of DS+A to lower proliferation (Gajewsky et al. 1988). However, IFN-gdoes not appear to have mediated the decrease in endogenous proliferation since a constant amount of IFN-g was present in the DS+A added to each culture but the amount of decrease in proliferation which occurred in response to DS+A varied. Also, the small but significant amount of IFN-gsecreted in response to antigen did not decrease proliferation when antigen was added.

The data from the CD4S experiments show that a factor secreted by Leishmania specific T1 CD4+ $\mathrm{T}$ cells derived from DTH+ adults was toxic or inhibitory to CD8+ T cells but not to CD4+ T cells. The effect was not mediated by IFN-g, tumor necrosis factor-a, or lymphotoxin. The factor in CD4S is similar to that in DS+A in that it might decrease the number of $\mathrm{CD} 8+$ Ts cells relative to $\mathrm{T} 1 \mathrm{CD} 4+\mathrm{T}$ cells. In fact the CD4S and DS+A factors might be the same. The CD4S data also suggest that the failure of DS+A to cause greater reduction in endogenous proliferation might be due to the fact that maximal inhibition or toxicity of the factors to $\mathrm{CD} 8+\mathrm{T}$ cells occurred three-four weeks after exposure to the factors rather than during the first five days when the DS+A proliferation assays were performed.

The data provide new insights into how antileishmania responses might be regulated in humans (Fig. 4). The model predicts that during L. chagasi infection Leishmania antigens prevent CD8+ Ts cells from secreting IL-10 and stimulate production of DS+A/CD4S factor(s) which decreases the number of $\mathrm{CD} 8+\mathrm{Ts}$ cells relative to $\mathrm{T} 1 \mathrm{CD} 4+\mathrm{T}$ 
cells. Leishmania specific T1 CD4+ T cells can then eradicate the infection. The resulting lack of Leishmania antigens allows CD8+ Ts cells to increase in number and secrete more IL-10. The CD8+ Ts cells can then prevent over-expansion and excess activity of $\mathrm{T} 1 \mathrm{CD} 4+\mathrm{T}$ cells from damaging the body and preventing other needed immune responses. Symptomatic infections occur when this immune regulatory cycle fails due to antagonistic demands on the immune system by concurrent infections, allergies, malnutrition, stress or other causes. The data suggest that Leishmania antigens and DS+A/CD4S factor(s) might be admistered to high activity patients to favor the development of $\mathrm{T} 1 \mathrm{CD} 4+\mathrm{T}$ cell responses which could eradicate their infections.

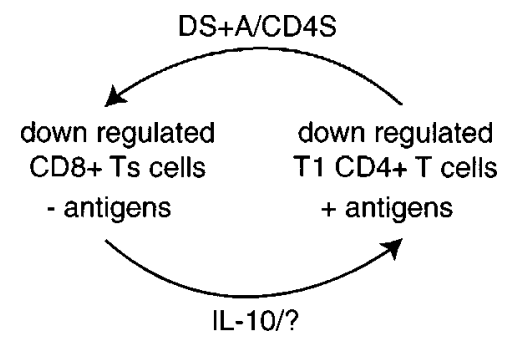

Fig. 4: a new model of cross regulation of human anti-leishmania responses showing the role of DS+A (supernatant generated from peripheral blood mononuclear cells from skin test positive adults by Leishmania antigen stimulation) and CD4S (supernatant derived by phytohemagglutinin stimulation of Leishmania specific $\mathrm{T} 1 \mathrm{CD} 4+\mathrm{T}$ cell clones derived from DTH+ adults) factor(s) in down regulating CD8+ Ts cells.

There is evidence to show that CD8+ Ts cells regulate $\mathrm{T} 1$ responses to other antigens in addition to $L$. chagasi antigens and that their activity is not always mediated by increased IL-10 secretion. Addition of acute phase CD8+ T cells to autologous PBMC isolated from $\mathrm{P} 2$ one year after successful treatment decreased IFN-gsecretion to other antigens in addition to L. chagasi antigens. The CD8+ T cells decreased IFN-gsecretion in response to Candida antigens from $2.23 \mathrm{ng} / \mathrm{ml}$ to $0 \mathrm{ng} / \mathrm{ml}$ and to tetanus toxoid antigens from $0.24 \mathrm{ng} / \mathrm{ml}$ to $0.06 \mathrm{ng} / \mathrm{ml}$. There was no antigen specific cytokine response to tuberculin but the CD8+ Ts cells reduced the proliferation SI to tuberculin from 6.5 to 1.3. There was no measurable IL-10 secretion in the presence or absence of the antigens in any of these assays. The ability of CD8+ Ts cells to inhibit responses to other antigens might explain the anergy of VL patients to non-leishmania antigens and most of the mortality of VL patients which is due to secondary infections (Veress et al. 1977, Aikat et al. 1979, Ho et al. 1983). Leishmania antigens and the DS+A/CD4S factor(s) might be used to prevent these deaths. The same approach might be useful in treating other infections in which Ts or T2 CD8+ T cells are implicated in immunopathology, such as leprosy and AIDS.

\section{ACKNOWLEDGMENTS}

To AQ Sousa, SMB Jeronimo and CH Costa for supplying blood samples and clinical data from VL patients; TG Evans and MJ Teixeira for identifying the DTH+ adults in this study; R Pearson, R Locksley and A Vasconcelos for laboratory facilities. To Genetics Institute for supplying rIL-12 and polyclonal anti-IL-12, to Hoffmann-La Roche for supplying monoclonal anti-IL12 , to Genentech for supplying anti-tumor necrosis factor-a and anti-lymphotoxin, to DNAX for supplying antiIL-10 and anti-IFN-gand to Cetus for supplying rIL-2.

\section{REFERENCES}

Aikat BK, Pathania AGS, Sehgal S, Bhattacharya PK, Dutta U, Pasricha N, Singh S, Parman RS, Sahaya S, Prasad LSN 1979. Immunological responses in Indian kala-azar. Indian J Med Res 70: 583-591.

Barcellini W, Rizzardi GP, Borghi MO, Fain C, Lazzarin A, Meroni PL 1994. TH1 and TH2 cytokine production by peripheral blood mononuclear cells from HIV-infected patients. AIDS 8: 757-762.

Berens R, Marr J 1978. An easily prepared defined medium for cultivation of Leishmania donovani promastigotes. J Parasitol 64: 160-162.

Carvalho E, Bacellar O, Brownell C, Regis T, Coffman RL, Reed SG 1994. Restoration of IFN-g production and lymphocyte proliferation in visceral leishmaniasis. J Immunol 152: 5949-5956.

Cenini P, Berhe N, Hailu A, McGinnes K, Frommel D 1993. Mononuclear cell subpopulations and cytokine levels in human visceral leishmaniasis before and after chemotherapy. J Infect Dis 168: 986-993.

Chomarat P, Rissoan M-C, Banchereau J, Miossec P 1993. Interferon-ginhibits interleukin 10 production by monocytes. J Exp Med 177: 523-527.

Convit J, Castellanos P, Ulrich M, Castes M, Rondon A, Pinardi ME, Rodriguez N, Bloom BR, Formica S, Valecillos L, Bretana A 1989. Immunotherapy of localized, intermediate, and diffuse forms of American cutaneous leishmaniasis. J Infect Dis 160: 104-115.

Convit J, Rondon A, Ulrich M, Bloom B, Castellano PL, Pinardi M E, Castes M, Garcia L 1987. Immunotherapy versus chemotherapy in localized cutaneous leishmaniasis. Lancet 1: 401-404.

Denis M, Ghadirian E 1994. Dysregulation of interleukin 8 , interleukin 10 , and interleukin 12 release by alveolar macrophages from HIV type 1 infected subjects. AIDS Res Hum Retroviruses 10: 1619-1627.

Dulley J, Grieve P 1975. A simple technique for eliminating interference by detergents in the Lowry method of protein determination. Anal Biochem 64: 136-141.

Evans T, Teixeira M, McAuliffe I, Vasconcelos I, Vasconcelos AW, Sousa AQ, Lima JW, Pearson RD 1992. Epidemiology of visceral leishmaniasis in northeast Brazil. J Infect Dis 166:1124-1132. 
Gajewski TF, Fitch FW 1988. Antiproliferative effect of IFN-gin immune regulation I. IFN-ginhibits proliferation of Th2 but not Th1 murine helper T lymphocyte clones. J Immunol 140: 4245.

Ghalib H, Piuvezam M, Skeiky Y 1993. Interleukin 10 production correlates with pathology in human Leishmania donovani infections. J Clin Invest 92: 324329.

Ghalib H, Whittle JA, Kubin M, Hashim FA, El-Hassan AM, Grabstein KH, Trinchieri B, Reed SG 1995. IL-12 enhances Th1-type responses in human Leishmania donovani infections. J Immunol 154:46234629.

Ho M, Koech DK, Iha DW, Bryceson ADM 1983. Immunosuppression in Kenyan visceral leishmaniasis. Clin Exp Immunol 51: 207-214.

Holaday BH, Pompeu MM, Jeronimo S, Texeira MJ, Sousa AQ, Vasconcelos AW, Pearson RD, Abrams JS, Locksley RM 1993. Potential role for interleukin10 in the immunosuppression associated with kala azar. J Clin Invest 92: 2626-2632.

Holaday BH, Pompeu MM, Evans T, Braga DN, Texeira MJ, Sousa AQ, Sadick MD, Vasconcelos AW, Abrams JS, Pearson RD, Locksley RM 1993. Correlates of Leishmania-specific immunity in the clinical spectrum of infection with Leishmania chagasi. J Infec Dis 167: 411-417.

Hsieh C-S, Macatonia S, Tripp C, Wolf SF, O'Garra A, Murphy KM 1993. Development of Th1 CD4+ T cells through IL-12 produced by Listeria-induced macrophages. Science 260: 547-549.

Karp CL, El-Safi SH, Wynn TA, Satti MMH, Kordofani AM, Hashim FA, Hag-Ali M, Neva FA, Nutman TB, Sacks DL 1993. In vivo cytokine profiles in patients with kala-azar: Marked elevation of both interleukin10 and interferon-gamma. J Clin Invest 91: 1644-
1648.

Maggi E, Giudizi MG, Biagiotti R, Annunziato F, Manetti R, Piccinni M-P, Parronchi P, Sampognaro S, Giannarini L, Zuccati G, Romagnani S 1994. Th2like CD8+ T cells showing B cell helper function and reduced cytolytic activity in human immunodeficiency virus type 1 infection. J Exp Med 180: 489495.

Manetti R, Parronchi P, Giudizi M, Piccinni M-P, Maggi E, Trinchieri G, Romagnani S 1993. Natural killer cell stimulatory factor [interleukin 12(IL-12)] induces $\mathrm{T}$ helper type 1 (Th1)-specific immune responses and inhibits the development of IL-4-producing Th cells. J Exp Med 177:1199-1204.

Salgame P, Abrams JS, Clayberger C, Goldstein H, Convit J, Modlin RL, Bloom BR 1991. Differing lymphokine profiles of functional subsets of human CD4 and CD8 T cell clones. Science 254: 279-282.

Uyemura K, Pirmez C, Sieling P, Kiene K, Paes-Oliveira M, Modlin RL 1993. CD4+ type 1 and CD8+ type $2 \mathrm{~T}$ cell subsets in human leishmaniasis have distinct $\mathrm{T}$ cell receptor repertoires. J Immunol 151: 7095-7104.

Veress B, Omer A, Satir AA, El Hassan AM 1977. Morphology of the spleen and lymph nodes in fatal visceral leishmaniasis. Immunology 33: 605-609.

Yssel H, de Vries J, Koken M, Blitterswijk WV, Spits H 1984. Serum-free medium for generation and propagation of functional human cytotoxic and helper $\mathrm{T}$ cell clones. J Immunol Methods 72: 219-227.

Zwingenberger K, Harms G, Pedrosa C, Omena S, Sandkamp B, Neifer S 1990. Determinants of the immune response in visceral leishmaniasis: Evidence for predominance of endogenous interleukin 4 over interferon-g production. Clin Immunol Immunopathol 57: 242-249. 